\title{
3D through silicon via profile metrology based on spectroscopic reflectometry for SOI applications
}

Fursenko, O., Bauer, J., Marschmeyer, S.

O. Fursenko, J. Bauer, S. Marschmeyer, "3D through silicon via profile metrology based on spectroscopic reflectometry for SOI applications," Proc. SPIE 9890, Optical Micro- and Nanometrology VI, 989015 (26 April 2016); doi: $10.1117 / 12.2227651$

SPIE. Event: SPIE Photonics Europe, 2016, Brussels, Belgium 


\title{
3D Through Silicon Via profile metrology based on spectroscopic reflectometry for SOI applications
}

\author{
O. Fursenko*a ${ }^{*}$ J. Bauer ${ }^{\mathrm{b}}$, S. Marschmeyer ${ }^{\mathrm{a}}$ \\ ${ }^{a}$ IHP, Im Technologiepark 25, 15236 Frankfurt (Oder), Germany; ${ }^{b}$ Technical University of \\ Applied Sciences, Hochschulring 1, 15745 Wildau, Germany
}

\begin{abstract}
Through-silicon via (TSV) technology is a key feature for 3D circuit integration. TSVs are formed by etching a vertical via and filling them with a conductive material for creation of interconnections which go through the silicon or siliconon-insulator (SOI) wafer. The Bosch etch process on Deep Reactive Ion Etching (DRIE) is commonly used for this purpose. The etch profile defined by the critical dimensions (CDs) at the top and at the bottom, by the depth and by the scallop size on the sidewall needs to be monitored and well controlled.

In this work a nondestructive 3D metrology of deeply-etched structures with an aspect ratio of more than 10 and patterns with lateral dimensions from 2 to $7 \mu \mathrm{m}$ in SOI wafer is proposed. Spectroscopic reflectometry in the spectral range of 250-800 $\mathrm{nm}$ using a production metrology tool was applied. The depth determinations based on different algorithms are compared. The Pearson correlation coefficient between measured and calculated reflection is suggested as the most appropriate method.

A simple method for top $\mathrm{CD}$ evaluation is proposed by the measurement of reflection and using the polynomial approximation of reflection versus TSV filling coefficient which is determined as ratio of CD to pitch. The 3D RCWA simulations confirm this dependence.
\end{abstract}

Keywords: spectroscopic reflectometry, through silicon via (TSV), SOI, etch depth, critical dimension (CD)

\section{INTRODUCTION}

Through Silicon Via (TSV) technology is a key feature of new 3D integration of micro-electro-mechanical systems (MEMS), photonic circuits, microfluidic devices, or electrical grounding of CMOS and BiCMOS devices [1-3]. TSVs are formed by etching a vertical via and filling them with a conductive material for creation of interconnections which go through the silicon or SOI wafer. Typically, the highly-selective Bosch Si etch process characterized by a high etch rate and high aspect ratio is used for via etching [4]. The etch profile defined by the critical dimensions (CDs) at the top and at the bottom, by the depth and by the scallop size (period and amplitude) needs to be monitored and well controlled to ensure all requirements for 3D interconnects. Usually, secondary electron microscopy (SEM) cross-section image analysis is applied. But SEM is destructive, time consuming and depends on the cutting technique. Optical interferometry and reflectometry are the main nondestructive metrological methods for this purpose [5-6] but their use is limited by smaller dimension and high aspect ratio due to the requirement of obtaining a measurable signal of reflected light from the bottom of the TSV.

We proposed [7, 8] a nondestructive 3D metrology of deeply-etched structures with an aspect ratio (AR) of more than 10 and patterns with lateral dimensions from 3 to $7 \mu \mathrm{m}$ on bulk Si wafers. Spectroscopic reflectometry in the spectral range of 250-800 $\mathrm{nm}$ using a production metrology tool was applied. The TSV depths were determined based on interference effect between waves reflected from TSV's top and bottom surfaces. The scallop size was estimated from the back diffraction effect of light from the side wall [8]. The rigorous coupled wave analysis (RCWA) was applied for top and bottom CDs evaluation and the scallop amplitude.

\footnotetext{
*fursenko@ihp-microelectronics.com
}

Optical Micro- and Nanometrology VI, edited by Christophe Gorecki, Anand Krishna Asundi, Wolfgang Osten, Proc. of SPIE Vol. 9890, 989015 - (C) 2016 SPIE · CCC code: 0277-786X/16/\$18 - doi: 10.1117/12.2227651 
In the current work we extended the method proposed in [7, 8] for the evaluation of TSV profiles (depth, CD) for SOI wafers. The use of SOI is mandatory in some high voltage applications. SOI wafers are a promising substrate for photonics circuits [9], MEMS manufacturing [10]. In case of SOI wafer the difficulties for depth determination can be observed due to overlapping of SOI and TSV interference patterns. Here, a stable algorithm for depth determination will be suggested. Additionally we will propose a simple method for top CD evaluation by the measurement of reflection and using the approximation of reflection versus TSV filling coefficient defined as the ratio between CD and pitch.

\section{EXPERIMENTAL}

In this work we consider high density TSV arrays in SOI wafer consisting of high aspect-ratio vias with a tapered profile and a nominal width (or CD) from 2 to $7 \mu \mathrm{m}$, a pitch in the range of 7-11 $\mu \mathrm{m}$, and etch depths of 40-60 $\mu \mathrm{m}$ (Table 1). The structures were manufactured by Deep Reactive Ion Etching (DRIE) with the Bosch process: isotropic dry etching with sulfur hexafluoride (SF6) and sidewall passivation by polymer deposition with octafluorocyclobutane and oxygen (C4F8/O2), in the tool Tegal 200 (SPTS). The SOI layer was $\sim 4 \mathrm{~m}$ thick on $1 \mu \mathrm{m}$ Buried Oxide (BOX).

The normal incidence reflectometry in the spectral range of 250-800 nm implemented in the wafer metrology tool KLA-Tencor Spectra Fx 200 was used for TSV characterizations. The optical objective with low numerical aperture (NA $<0.01$, angle of incidence $\alpha \sim 88.23^{\circ}$ ) and an illumination spot size of about $40 \mu \mathrm{m}$ was utilized. The wavelengths resolution was $\sim 1.25 \mathrm{~nm}$. TSV depth was extracted from the interference effect between waves reflected from TSV's top and bottom surfaces [7,8]. The rigorous coupled wave analysis (RCWA) based on light scattering with the commercial simulation software [11] is applied for TSV top and bottom CD. Scanning electron microscopy (SEM) inspections were used in order to prove our results.

\section{RESULTS AND DISCUSSION}

Fig.1 shows a SEM view of the investigated TSV array structures. Two types of surface texture can be observed on the TSV side walls formed by DRIE in Si. The first type is the scalloping effect which is caused by the alternating isotropic etch and passivation steps in the Bosch process. The second type is a random sidewall roughness due to the ion behavior during etching. Wall scalloping occurs primarily in the top region where scattered ions have wide trajectories, and decrease with depths where ion trajectories are more restricted.
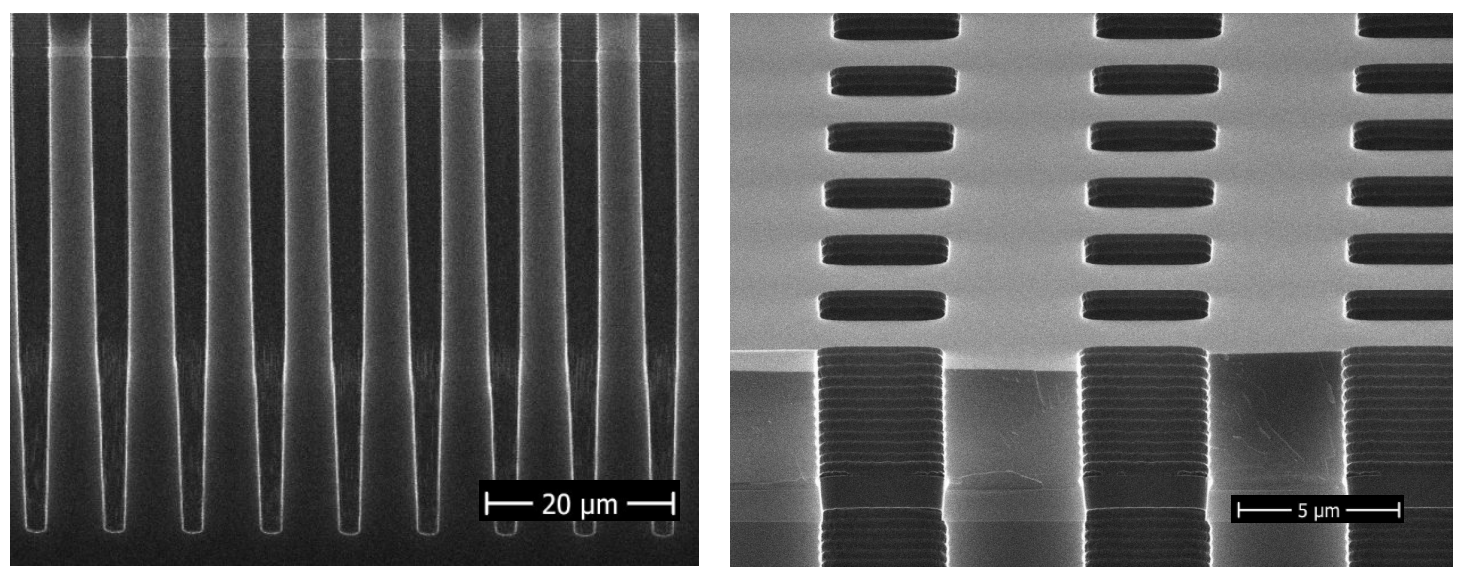

Fig. 1. SEM cross sections of TSV 2 arrays (Table 1) in SOI wafer. 
The reflection spectra for different TSV structures etched in the same wafer with various CDs (Table 1) are presented in Fig. 2 a. The reflectance spectra oscillate at distinct frequencies over the wavelength range of $\sim 400-800$ $\mathrm{nm}$. The high frequency oscillations are the consequence of the interference effect between the reflected waves from the top and bottom surfaces of the TSV structures, whereas the low frequency oscillations for $\lambda>500 \mathrm{~nm}$ are caused by the interference effect produced by SOI. The wavelength range of such oscillations is defined by SOI thickness.

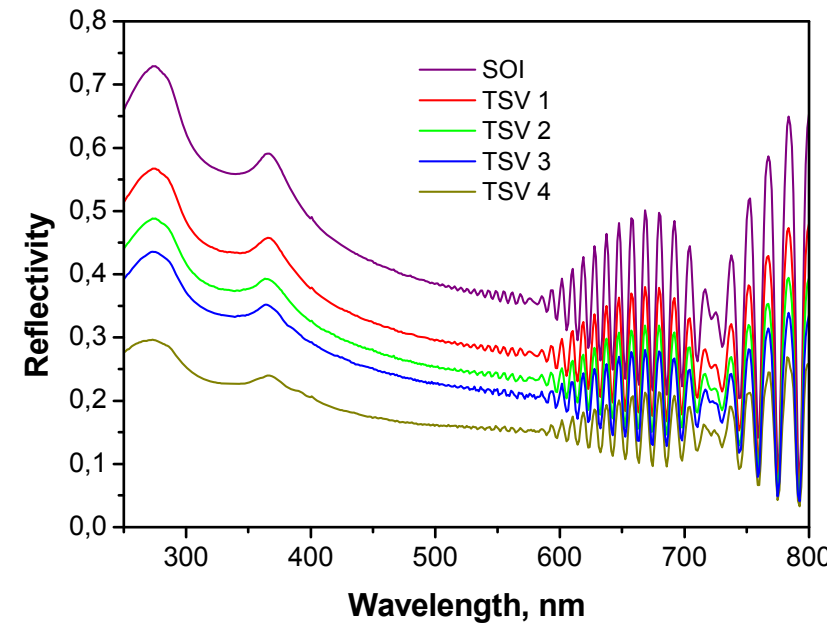

a)

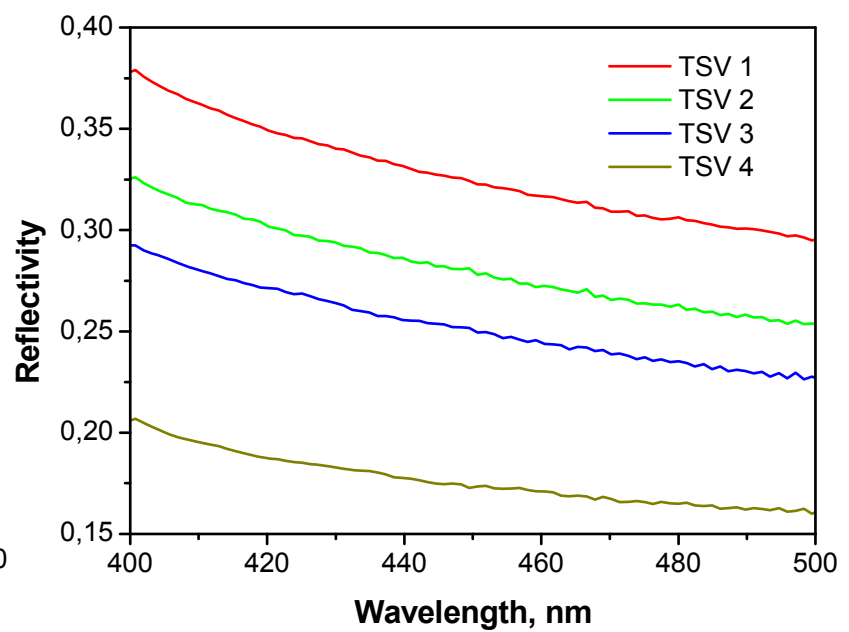

b)

Fig. 2. Experimental reflection spectra for different CDs of TSVs (Table 1) etched in SOI wafer.

For the case of $4 \mu \mathrm{m}$ SOI an overlap of SOI and TSV interference patterns is observed in the spectral range above $\sim 500 \mathrm{~nm}$. Therefore, for TSV profile evaluation only a limited wavelength range $(\lambda=400-500 \mathrm{~nm})$ with weak interference patterns from TSVs is used (Fig. 2 b). The beginning of such peculiarity at $\lambda \sim 400 \mathrm{~nm}$ is due to the relative low spectral resolution of the used metrology tool and the subsequent smaller coherence length $\left(\Delta l=\lambda^{2} / \Delta \lambda\right)$ in the short wavelength range. The TSV depth can be determined from the period of the high frequency oscillations of the reflection (R). The simple calculation procedure was described in [7].

We have experimentally compared several common algorithms in terms of solvability, correctness, uniqueness, reliability, running time and robustness. The main problem encountered by all methods is to find the unique mathematical solution for via depth determination from the distorted optical interference signals (Fig. 2 b). Three algorithms have been considered: least squares minimization of the sum of squares differences between measured and simulated reflection, which is previously presented for bulk Si wafer in [7], Fourier transformation of reflection and Pearson correlation coefficient between measured and simulated reflection. The experimental results from running the different optimization algorithms are given in Fig. 3. We observed, that the method for depth determination based on the least squares minimization of the sum of squares differences between measured and simulated reflection (Fig. 3, a) [7] cannot be used for low lateral TSV dimensions (in our case for $\mathrm{CD}<4 \mu \mathrm{m}$ ) for SOI wafer where the computing of the global minimum is impossible. Fitting difficulties for TSVs with small CDs are associated with the small coherence length, the low back reflection signal from the TSV bottom and caused by insufficient models of reflection from the TSV structures. For the Fourier transformation approach (Fig. 3, b), used also by $\mathrm{Ku}$ [6], unique mathematical solutions for the TSV depth evaluation are possible, too, due to high sensitivity of the Fourier transformation frequency analysis. Only difficulties can be produces by the additional peaks appear for TSV depths smaller than $40 \mu \mathrm{m}$ due to reflection oscillations by SOI. The relative low spectral resolution of the used metrology tool can decrease the depth resolution up to $1 \mu \mathrm{m}$. The best optimization can be performed by calculation of the maximum of Pearson correlation coefficient between measured and calculated reflection (Fig. 3, c). The method is shown to be effective and computationally compact to have high convergence speed and robustness. Here, we found a good sensitivity to the interference 
oscillations between waves reflected from TSV's top and bottom surfaces with less influence of reflection by SOI layers. Using this algorithm we achieved results which are extremely close to the global minimum with less than 1 percent error. The depth determination is reliable for all investigated CDs.

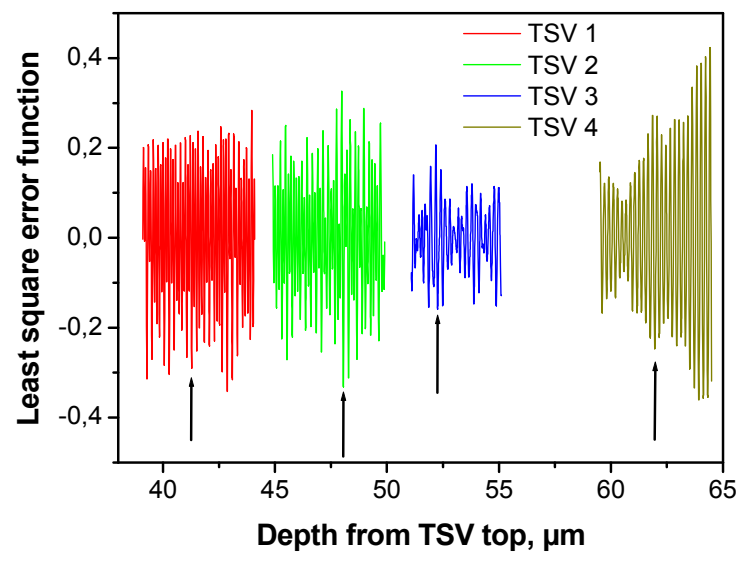

a)

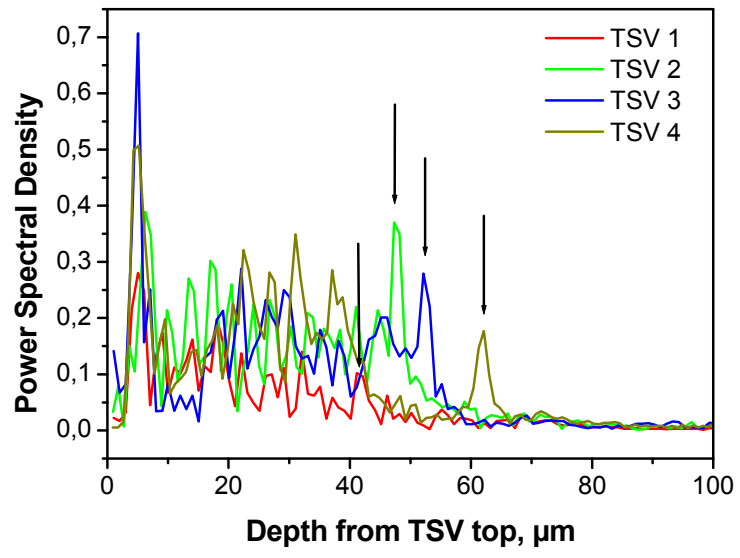

b)

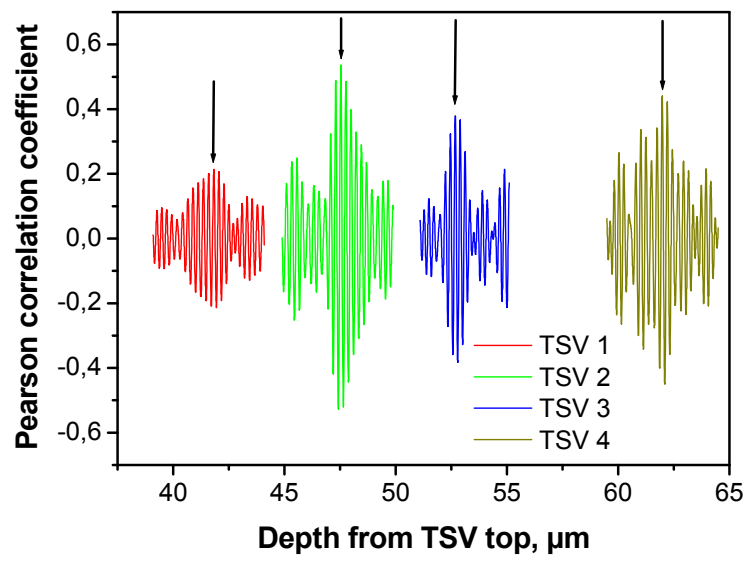

c)

Fig. 3. Performance of 3 optimization algorithms: least squares minimization of the sum of squares differences between measured and simulated reflection (a), Fourier transformation of reflection (b) and Pearson correlation coefficient between measured and simulated reflection (c). Arrows define the structure depth.

The calculation precision obtained for a set of different CDs allows one an effective control of etch rate. The accuracy increases with increasing feature size and decreasing AR. Fig. 4 shows a very good TSV depth uniformity over the wafer with less than $2 \%$ depth variation for minimal CD $(2.5 \mu \mathrm{m})$ over the wafer. TSV depth determination with the described fitting procedure is possible across a wide range of TSV CDs and ARs (Table 1). 

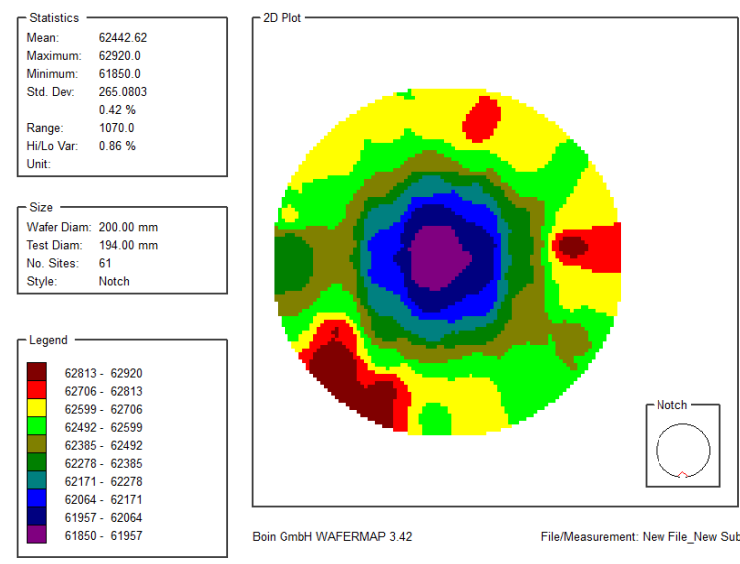

Boin GmbH WAFERMAP 3.42

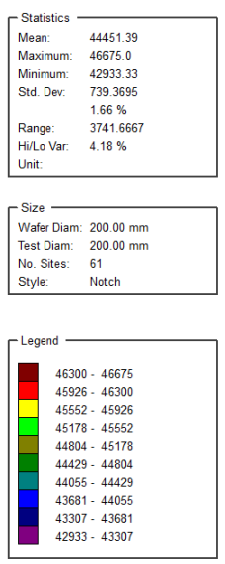

a)

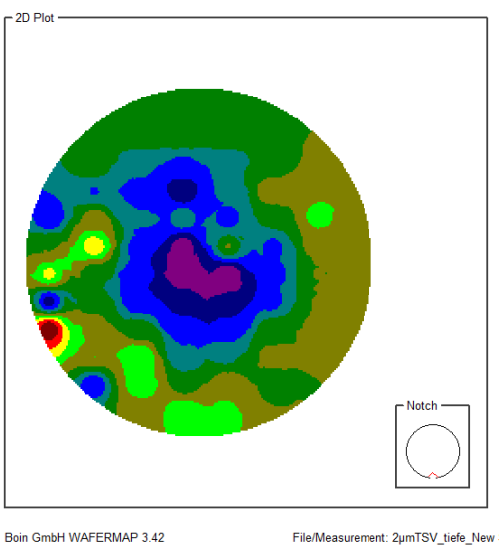

b)

Fig. 4. TSV depth map for TSV 1 (a) and TSV 4 (b) structures in SOI wafer.

The TSV top and bottom CD evaluation can be realized by RCWA based on the scattered light from a periodic array [11] as we have shown in [7,8]. However, this method is time consuming. Here we propose a simple method for top CD evaluation by the measurement of reflection and using the third-order polynomial fit of reflection versus TSV filling coefficient $\left(f_{v i a}\right)$ defined as the ratio between CD and pitch (P) (Fig. 5). The polynomial fit (Fig. 5) is based on $600 \mathrm{CD}$ measurements of TSVs as well as of grating structures in SOI and in Si wafers. A $3 \sigma$ accuracy of $5 \%$ for CD evaluation can be reach. The 3D RCWA simulations confirm this dependence.

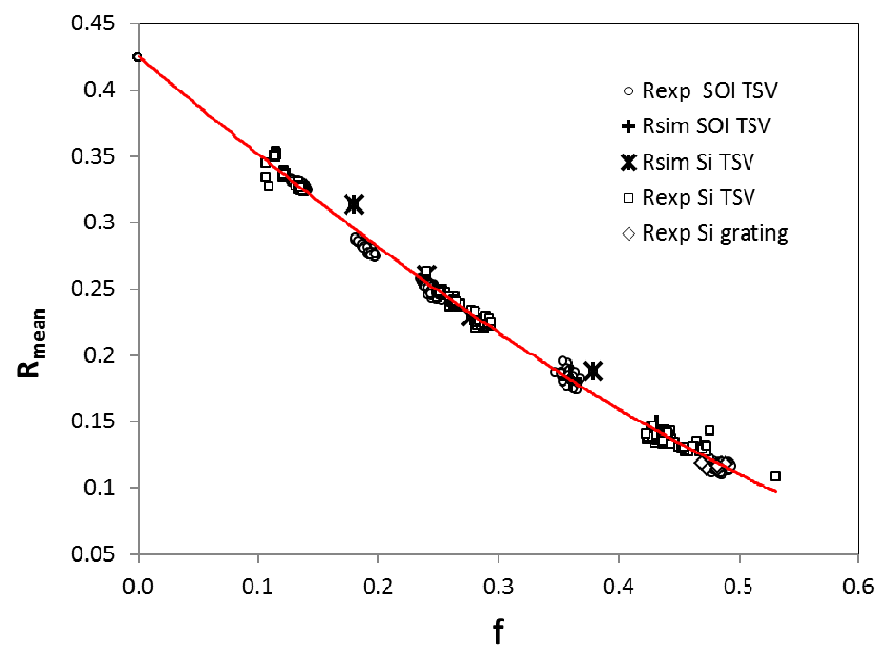

Fig. 5. Mean experimental and simulated reflectivity for $\lambda=400-500 \mathrm{~nm}$ vs. TSV filling coefficient $f_{v i a}=\left(C D_{x} C D_{y}\right) /\left(P_{x} P_{y}\right)$ for SOI wafer. Additionally, the results for TSV in bulk Si and for Si gratings $\left(f_{\text {grating }}=C D / P\right)$ are presented. The polynomial fit $\left(R_{\text {mean }}=0.263 f^{3}+0.114 f^{2}-0.752 f+0.425\right)$ is shown by line.

The results for etch depth, top and bottom CD of a single TSV structures in SOI wafer were compared with SEM cross section images and summarized in Table 1. 
Table 1. Geometrical parameters of a single TSV structures in SOI wafer obtained by spectral reflection (R) in comparison with SEM results.

\begin{tabular}{|c|c|c|c|c|c|c|c|c|}
\hline \multirow[t]{2}{*}{ Sample } & \multicolumn{2}{|c|}{1} & \multicolumn{2}{|c|}{2} & \multicolumn{2}{|c|}{3} & \multicolumn{2}{|c|}{4} \\
\hline & 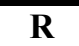 & 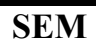 & $\overline{\mathbf{R}}$ & $\bar{~} \overline{\text { SEM }}$ & 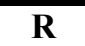 & 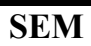 & 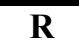 & $\overline{\text { SEM }}$ \\
\hline "Top CDx, $\mu \mathrm{m}$ & 2.48 & 2.53 & 3.27 & 3.21 & 3.72 & 3.67 & 6.23 & 6.23 \\
\hline Top CDy, $\mu \mathrm{m}$ & 2.56 & 2.62 & 3.18 & 3.12 & 4.63 & 4.77 & 6.81 & 6.82 \\
\hline $\operatorname{Pitch} x, \mu \mathrm{m}$ & \multicolumn{2}{|c|}{7.4} & \multicolumn{2}{|c|}{7.4} & \multicolumn{2}{|c|}{8} & \multicolumn{2}{|c|}{10.7} \\
\hline Pitch $y, \mu m$ & \multicolumn{2}{|c|}{7} & \multicolumn{2}{|c|}{7.5} & \multicolumn{2}{|c|}{9.2} & \multicolumn{2}{|c|}{11.2} \\
\hline Depth, $\mu \mathrm{m}$ & 41.8 & 41.6 & 47.5 & 48.4 & 52.7 & 53.9 & 62 & 61.9 \\
\hline
\end{tabular}

\section{CONCLUSION}

3D TSV profile control (depth, critical dimensions (CD)) of Bosch etching process in SOI substrate was performed by spectroscopic reflectometry in the 200-800 nm wavelength range. Based on interference and diffraction effects the etch profile of TSV structures with an aspect ratio of up to 17 and patterns with CDs from 2.5 to $7 \mu \mathrm{m}$ in SOI wafers was evaluated. The depth determinations based on different minimization algorithms were compared. The effective approach based on calculation of maximum of Pearson correlation coefficient between measured and calculated reflection was suggested. TSV depth determination error is about $1 \%$ for $\mathrm{CD} \gtrsim 4 \mu \mathrm{m}$.

A simple method for top CD evaluation (with $5 \%$ error $(3 \sigma)$ ) by the $\mathrm{R}$ measurements was proposed and confirmed by 3D RCWA simulations.

Nondestructive metrology monitoring using R measurements provide excellent correlation with SEM results.

The stable 3D TSV profile metrology by spectroscopic reflectometry for SOI application was realized. Such inline non-destructive metrology offers significant advantages in TSV etch process development and in reducing dependency on destructive characterization techniques such as cross section SEM. This method can be utilized for the evaluation of chip-to-chip and wafer-to-wafer variations in the volume manufacturing.

\section{REFERENCES}

[1] Gerke, D.,[NASA 2009 Body of Knowledge (BoK): Through-Silicon via Technology], JPL Publication 9-28, (2009).

[2] Wietstruck, M., Kaynak, M., Marschmeyer, St., Wipf, C., Tekin, I., Zoschke, K., and Tillack, B., "Modeling and Optimization of BiCMOS Embedded Through-Silicon Vias for RF-Grounding", Proc. 14th Topical Meeting on Silicon Monolithic Integrated Circuits in RF Systems (SiRF 2014), 83 (2014).

[3] Marschmeyer, S., Berthold, J., Krüger, A., Lisker, M., Scheit, A., Schulze, S., Trusch, A., Wietstruck, M., Wolansky, D., "Modular integration of annular TSV structures filled with tungsten in a $0.25 \mu \mathrm{m} \mathrm{SiGe:C}$ BiCMOS Technology", Microelectron. Eng. 137, 153-157 (2015).

[4] Laermer, F. and Schilp, A., "Method for anisotropically etching silicon", German patent DE4241045 (1992).

[5] Vartanian, V., Allen, R.A., Smith, L., Hummler, K., Olson, S., Sapp, B., "Metrology needs for through-silicon via fabrication", J. Micro/Nanolith. MEMS MOEMS 13 (1), 011206 (2014).

[6] Ku, Y.S., Huang, K.C., Hsu, W., "Characterization of high density through silicon vias with spectral reflectometry", Opt. Express 19 (7), 5993-6006 (2011).

[7] Fursenko, O., Bauer, J., Marschmeyer, S., "In-line through silicon vias etching depths inspection by spectroscopic Reflectometry", Microelectronic Engineering 122, 25-28 (2014).

[8] Fursenko, O., Bauer, J., Marschmeyer, S., Stoll, H.-P., "Through silicon via profile metrology of Bosch etching process based on spectroscopic reflectometry", Microelectronic Engineering 139, 70-75 (2015).

[9] Yang, Yan at al., "Through-Si-via (TSV) keep-out-zone (KOZ) in SOI photonics interposer: a study of the impact of TSV-induced stress on Si ring resonators", IEEE Photonics Journal 5(6), 2700611 (2013).

[10] Lietaer, Nicolas at al., "Wafer-level packaged MEMS switch with TSV", European Nanoelectronics forum, JEMSIP_3D, Nov.16-17, Madrid, Spain (2010).

[11] www.unigit.com 